\title{
Unicity of Meromorphic Solutions of Some Nonlinear Difference Equations
}

\section{Baoqin Chen}

Faculty of Mathematics and Computer Science, Guangdong Ocean University, Zhangjiang, China

Email: chenbaoqin_chbq@126.com

How to cite this paper: Chen, B.Q. (2019) Unicity of Meromorphic Solutions of Some Nonlinear Difference Equations. Advances in Pure Mathematics, 9, 611-618. https://doi.org/10.4236/apm.2019.97030

Received: June 18, 2019

Accepted: July 22, 2019

Published: July 25, 2019

Copyright (C) 2019 by author(s) and Scientific Research Publishing Inc. This work is licensed under the Creative Commons Attribution International License (CC BY 4.0).

http://creativecommons.org/licenses/by/4.0/

\begin{abstract}
This paper is to study the unicity of transcendental meromorphic solutions to some nonlinear difference equations. Let $m \in\{ \pm 2, \pm 1,0\}$ be a nonzero rational function. Consider the uniqueness of transcendental meromorphic solutions to some nonlinear difference equations of the form

$w(z+1) w(z-1)=R(z) w^{m}(z)$. For two finite order transcendental meromorphic solutions of the equation above, it shows that they are almost equal to each other except for a nonconstant factor, if they have the same zeros and poles counting multiplicities, when $m \in\{2, \pm 1,0\}$. Two relative results are proved, and examples to show sharpness of our results are provided.
\end{abstract}

\section{Keywords}

Unicity, Meromorphic Solution, Difference Equation

\section{Introduction}

It is well known that a given nonconstant monic polynomial is determined by its zeros. But it is not true for transcendental entire or meromorphic functions. Take $\mathrm{e}^{z}$ and $\mathrm{e}^{-z}$ for example, they are essentially different even have the same zeros, 1-value points and poles. This indicates that it is complex and interesting to determine a transcendental meromorphic function uniquely. Nevanlinna then proves his famous Nevanlinna's 5 CM (4 IM) Theorem (see e.g. [1] [2]):

Theorem A: Let $w(z)$ and $u(z)$ be two nonconstant meromorphic functions. If $w(z)$ and $u(z)$ share 5 values IM (4 values CM, respectively) in the extended complex plane, then $w(z) \equiv u(z)(w(z)=T(u(z)))$, where $T$ is a Möbius transformation, respectively).

Here and in the following, for two nonconstant meromorphic functions $w(z)$ 
and $u(z)$, and a complex constant $a$, we say $w(z)$ and $u(z)$ share a IM (CM), if $w(z)-a$ and $u(z)$-a have the same zeros ignoring multiplicities (counting multiplicities); and we say $w(z)$ and $u(z)$ share $\infty \operatorname{IM}(\mathrm{CM})$, if they have the same poles ignoring multiplicities (counting multiplicities).

Our aim is to study the unicity of meromorphic solutions to the nonlinear difference equation of the form

$$
w(z+1) w(z-1)=R(z) w^{m}(z),
$$

where $R(z)$ is a nonzero rational function and $m \in\{ \pm 2, \pm 1,0\}$ The Equation (1.1) comes from the family of Painlevé III equations which are given by Ronkainen in [3] when he classifies the difference equation

$$
w(z+1) w(z-1)=R(z, w),
$$

where $R(z, w)$ is irreducible and rational in $w$ and meromorphic in $z$. This is a natural idea which comes from the topic on the growth, value distribution and unicity on the meromorphic solutions to difference equations (see e.g. [4] [5] [6] [7] [8]). The first result is as follows.

Theorem 1.1. Let $w(z)$ and $u(z)$ be two finite order transcendental meromorphic solutions to the Equation (1.1), where $m \in\{2, \pm 1,0\}$. If $w(z)$ and $u(z)$ share $0, \infty \mathrm{CM}$, then $w(z) \equiv \lambda u(z)$, where $\lambda$ is a constant such that $\lambda^{2-m}=1$.

The following examples show that all cases in Theorem 1.1. can happen, and the "CM" cannot be relaxed to "IM".

Example 1. In the following examples, $w_{j}(z)$ and $u_{j}(z)$ share $0, \infty \mathrm{CM}$, while $w_{j}(z)$ and $v_{j}(z)$ share $0, \infty \operatorname{IM}(j=1,2,3,4)$ :

1) $u_{1}(z)=\tan \left(\frac{\pi z}{2}\right), w_{1}(z)=i u_{1}(z)$ and $v_{1}(z)=u_{1}^{2}(z)$ satisfy the difference equation

$$
w(z+1) w(z-1)=w^{-2}(z) .
$$

here $m=-2, \lambda=i$ such that $\lambda^{2-(-2)}=1$.

2) $u_{2}(z)=\tan ^{2}\left(\frac{\pi z}{3}\right) \tan ^{2}\left[\frac{(2 z-1) \pi}{6}\right], w_{2}(z)=\mathrm{e}^{i \frac{2 \pi}{5}} u_{2}(z)$ and $v_{2}(z)=u_{2}^{2}(z)$ satisfy the difference equation

$$
w(z+1) w(z-1)=w^{-1}(z)
$$

here $m=-1, \lambda=\mathrm{e}^{\frac{2 \pi i}{3}}$ such that $\lambda^{2-(-1)}=1$.

3) $u_{3}(z)=\tan \left(\frac{\pi z}{4}\right), w_{3}(z)=-u_{3}(z)$ and $v_{3}(z)=i u_{3}^{2}(z)$ satisfy the difference equation

$$
w(z+1) w(z-1)=-1 .
$$

here $m=0, \lambda=-1$ such that $\lambda^{2-0}=1$.

4) $u_{4}(z)=\tan \left(\frac{\pi z}{6}\right) \tan \left[\frac{\pi(z-1)}{6}\right], w_{4}(z)=u_{4}(z)$ and $v_{4}(z)=u_{4}^{3}(z)$ satisfy 
the difference equation

$$
w(z+1) w(z-1)=-w(z) .
$$

here $m=1, \lambda=1$ such that $\lambda^{2-1}=1$.

Theorem 1.2. Let $w(z)$ and $u(z)$ be two finite order transcendental meromorphic solutions to the Equation (1.1), where $m \in\{2, \pm 1,0\}$. If $w(z)$ and $u(z)$ share 0 , $\infty \mathrm{CM}$, then

$$
w(z) \equiv \mathrm{e}^{a_{2} z^{2}+a_{1} z+a_{0}} u(z),
$$

where $a_{0}, a_{1}, a_{2}$ are constants such that $\mathrm{e}^{2 a_{2}}=1$. What is more, $w(z) \equiv u(z)$ if $w(z)-u(z)$ has a zero $z_{1}$ of multiplicity $\geq 3$ such that $w\left(z_{1}\right)=u\left(z_{1}\right)=c \neq 0$.

The following example shows that all conclusions in Theorem 1.2 can happen, and the "CM" cannot be relaxed to "IM".

Example 2. Let $u(z)=\tan (\pi z), v(z)=u^{2}(z)$ and $w_{1}(z)=\mathrm{e}^{\pi i z^{2}} u(z)$, $w_{2}(z)=\mathrm{e}^{z} u(z), w_{3}(z)=u(z)$. Then $w_{j}(z)$ and $u(z)$ share $0, \infty \mathrm{CM}$, while $w_{j}(z)$ and $v(z)$ share $0, \infty \operatorname{IM}(j=1,2,3)$, and they solve the equation

$$
w(z+1) w(z-1)=w^{2}(z) .
$$

Theorem 1.3. Let $w(z)$ and $u(z)$ be two finite order transcendental meromorphic solutions to the Equation (1.1), where $m \in\{ \pm 1,0\}$. If $w(z)$ and $u(z)$ share $1, \infty \mathrm{CM}$, then

$$
w(z)-1 \equiv \mathrm{e}^{a_{1} z+a_{0}}(u(z)-1),
$$

where $a_{0}, a_{1}$ are constants such that:

1) $a_{1}=\frac{k_{1}}{2} \pi i$, when $m=0$;2) $a_{1}=\frac{2 k_{2}}{3} \pi i$, when $m=-1$; (3) $a_{1}=\frac{k_{3}}{3} \pi i$, when

$m=1$, where $k_{1}, k_{2}, k_{3}$ are some integers. What is more, $w(z) \equiv u(z)$ if one of the following additional condition holds:

a) $w(z)-u(z)$ has a zero $z_{1}$ of multiplicity $\geq 2$ such that $w\left(z_{1}\right)=u\left(z_{1}\right)=0$;

b) there exist two constants $z_{2}, z_{3}$ such that $w\left(z_{j}\right)=u\left(z_{j}\right) \neq 1(j=2,3)$ and $z_{2}-z_{3} \notin \mathbb{Q}$.

Remark 1. We have tried hard but failed to provide some similar results as Theorem 1.3 for the cases $m= \pm 2$ so far.

\section{Proof of Theorem 1.1}

Since $w(z)$ and $u(z)$ are finite order transcendental meromorphic functions and share $0, \infty \mathrm{CM}$, we see that

$$
\frac{w(z)}{u(z)}=\mathrm{e}^{p(z)},
$$

where $p(z)$ is a polynomial such that it is of degree

$\operatorname{deg} p(z)=p \leq \max \{\rho(w), \rho(u)\}$. 
Next, we discuss case by case.

Case 1: $m=-2$. From (1.1) and (2.1) we get

$$
\begin{aligned}
& u(z+1) u(z-1) u^{2}(z) \mathrm{e}^{p(z+1)+p(z-1)+2 p(z)} \\
& =w(z+1) w(z-1) w^{2}(z)=R(z)=u(z+1) u(z-1) u^{2}(z),
\end{aligned}
$$

which gives

$$
\left(\mathrm{e}^{p(z+1)+p(z-1)+2 p(z)}-1\right) u(z+1) u(z-1) u^{2}(z) \equiv 0 .
$$

Thus, we have

$$
\mathrm{e}^{p(z+1)+p(z-1)+2 p(z)} \equiv 1 .
$$

Since

$$
\operatorname{deg}(p(z+1)+p(z-1)+2 p(z))=\operatorname{deg} p(z)=p,
$$

from (2.2), it is easy to find that $p=0$. Therefore, there exists some constant $p_{0}$, such that $p(z) \equiv p_{0}$ and

$$
\mathrm{e}^{4 p_{0}}=\mathrm{e}^{p(z+1)+p(z-1)+2 p(z)} \equiv 1 .
$$

That is, for $\lambda=\mathrm{e}^{p_{0}}$, we have $w(z) \equiv \lambda u(z)$ and $\lambda^{4}=1$.

Case 2: $\boldsymbol{m}=-\mathbf{1}$. Now, we obtain from (1.1) and (2.1) that

$$
\begin{aligned}
& u(z+1) u(z-1) u(z) \mathrm{e}^{p(z+1)+p(z-1)+2 p(z)} \\
& =w(z+1) w(z-1) w(z)=R(z)=u(z+1) u(z-1) u(z) .
\end{aligned}
$$

With this equation and similar reasoning as in Case 1 , we can deduce that $w(z) \equiv \lambda u(z)$ holds for some $\lambda$ such that $\lambda^{3}=1$.

Case 3: $\boldsymbol{m}=\mathbf{0}$. From (1.1) and (2.1), we have

$$
u(z+1) u(z-1) \mathrm{e}^{p(z+1)+p(z-1)}=w(z+1) w(z-1)=R(z)=u(z+1) u(z-1) .
$$

Similarly, we can prove that $w(z) \equiv \lambda u(z)$ holds for some $\lambda$ such that $\lambda^{2}=1$.

Case 4: $\boldsymbol{m}=1$. Now (1.1) is of the form

$$
w(z+1) w(z-1)=R(z) w(z) .
$$

Thus,

$$
w(z+2) w(z)=R(z+1) w(z+1) .
$$

It follows from these two equations above and (2.1) that

$$
\begin{aligned}
& u(z+2) u(z-1) \mathrm{e}^{p(z+2)+p(z-1)} \\
& =w(z+2) w(z-1)=R(z+1) R(z)=u(z+2) u(z-1),
\end{aligned}
$$

with which we can show that $w(z) \equiv \lambda u(z)$ holds for some $\lambda$ such that $\lambda^{2}=1$. However, if $w(z) \equiv-u(z)$, we find that

$$
(-w(z+1))(-w(z-1))=u(z+1) u(z-1)=R(z) u(z)=-R(z) w(z) .
$$

Combining (2.3) and (2.4), we get $R(z) w(z) \equiv 0$, which is impossible. Thus, $\lambda=1$. 


\section{Proof of Theorem 1.2}

Notice that (2.1) still holds for this case. We can get from (1.1) and (2.1) that

$$
\frac{u(z+1) u(z-1) \mathrm{e}^{p(z+1)+p(z-1)}}{u^{2}(z) \mathrm{e}^{2 p(z)}}=\frac{w(z+1) w(z-1)}{w^{2}(z)}=R(z)=\frac{u(z+1) u(z-1)}{u^{2}(z)} .
$$

Thus, we have

$$
\mathrm{e}^{p(z+1)+p(z-1)+2 p(z)} \equiv 1 .
$$

If $p \leq 1$, then our conclusion holds for $a_{2}=0$. If $p \geq 2$, set

$$
p(z)=a_{p} z^{p}+a_{p-1} z^{p-1}+\cdots+a_{1} z+a_{0},
$$

where $a_{p} \neq 0, a_{p-1}, \cdots, a_{1}, a_{0}$ are constants.

From (3.2), we see that

$$
p(z+1)+p(z-1)-2 p(z)=p(p-1) a_{p} z^{p-2}+q(z),
$$

where $q(z)$ is a polynomial such that $q(z) \equiv 0$ when $p=2$, or $\operatorname{deg} q(z)<p-2$ when $p \geq 3$.

Suppose that $p \geq 3$, we obtain from (3.1) and (3.3) that

$$
1 \equiv \mathrm{e}^{p(z+1)+p(z-1)+2 p(z)}=\mathrm{e}^{p(p-1) a_{p} z^{p-2}+q(z)},
$$

which is impossible. Thus, $p=2$, then from (3.1) and (3.3), we get $\mathrm{e}^{2 a_{2}}=1$ immediately. To sum up, we prove that (1.2) holds.

Next, we use $p(z)=a_{2} z^{2}+a_{1} z+a_{0}$ and prove our additional conclusion. From (1.2), we see that $\mathrm{e}^{p\left(z_{1}\right)}=1$.

Differentiating both sides of (1.2), we can deduce that

$$
p^{\prime}(z) \mathrm{e}^{p(z)} u(z)=w^{\prime}(z)-\mathrm{e}^{p(z)} u^{\prime}(z)
$$

and

$$
p^{\prime \prime}(z) \mathrm{e}^{p(z)} u(z)=w^{\prime \prime}(z) \mathrm{e}^{p(z)} u(z)=\left(p^{\prime}(z)\right)^{2} \mathrm{e}^{p(z)} u(z)-2 p^{\prime}(z) \mathrm{e}^{p(z)} u^{\prime}(z) .
$$

By our assumption, (1.2\}), (3.4) and the fact that $\mathrm{e}^{p\left(z_{1}\right)}=1$, we have

$$
\begin{aligned}
p^{\prime}\left(z_{1}\right) & =p^{\prime}\left(z_{1}\right) u\left(z_{1}\right)=p^{\prime}\left(z_{1}\right) \mathrm{e}^{p\left(z_{1}\right)} u\left(z_{1}\right) \\
& =w^{\prime}\left(z_{1}\right)-\mathrm{e}^{p\left(z_{1}\right)} u^{\prime}\left(z_{1}\right) \\
& =w^{\prime}\left(z_{1}\right)-u^{\prime}\left(z_{1}\right)=0 .
\end{aligned}
$$

Therefore, similarly, it follows from (3.5) that

$$
\begin{aligned}
p^{\prime \prime}\left(z_{1}\right) & =p^{\prime \prime}\left(z_{1}\right) \mathrm{e}^{p\left(z_{1}\right)} u\left(z_{1}\right) \\
& =w^{\prime \prime}\left(z_{1}\right)-\mathrm{e}^{p\left(z_{1}\right)} u^{\prime \prime}\left(z_{1}\right)-\left(p^{\prime}\left(z_{1}\right)\right)^{2} \mathrm{e}^{p\left(z_{1}\right)} u\left(z_{1}\right)-2 p^{\prime}\left(z_{1}\right) \mathrm{e}^{p\left(z_{1}\right)} u^{\prime}\left(z_{1}\right) \\
& =w^{\prime \prime}\left(z_{1}\right)-u^{\prime \prime}\left(z_{1}\right)=0 .
\end{aligned}
$$

As a result, we obtain

$$
2 a_{2}=p^{\prime \prime}\left(z_{1}\right)=0,2 a_{2} z_{1}+a_{1}=p^{\prime}\left(z_{1}\right)=0, \mathrm{e}^{2 a_{2} z_{1}^{2}+a_{0} z_{1}+a_{0}}=\mathrm{e}^{p\left(z_{1}\right)}=1,
$$

that is, $a_{2}=a_{1}=0, \mathrm{e}^{a_{0}}=1$. Hence, $w(z) \equiv \mathrm{e}^{a_{2} z^{2}+a_{1} z+a_{0}} u(z)=u(z)$. 


\section{Proof of Theorem 1.3}

Here, we need the lemma below, where the case that $R(z)$ is a nonzero constant has been proved by Zhang and Yang [7] and the case that $R(z)$ is a nonconstant rational function by Lan and Chen [8].

Lemma 4.1. [7] [8] Let $w(z)$ be a finite order transcendental meromorphic solution to

the Equation (1.1), where $m \in\{-2, \pm 1,0\}$ and $a$ be a constant. Then

$$
\lambda(w-a)=\lambda(1 / w)=\rho(w) \geq 1 .
$$

Proof of Theorem 1.3. Since $w(z)$ and $u(z)$ are finite order transcendental meromorphic functions and share $1, \infty \mathrm{CM}$, we see that

$$
\frac{w(z)-1}{u(z)-1}=\mathrm{e}^{p(z)}
$$

where $p(z)$ is a polynomial such that

$$
p(z)=a_{p} z^{p}+a_{p-1} z^{p-1}+\cdots+a_{0},
$$

where $a_{p} \neq 0, \cdots, a_{0}$ are constants and $p=\operatorname{deg} p(z) \leq \max \{\rho(w), \rho(u)\}$.

Case 1: $\boldsymbol{m}=\mathbf{0}$. From (1.1) and (4.1), we obtain

$$
\frac{u(z+4)}{u(z)}=\frac{R(z+3)}{R(z+1)}:=R_{1}(z)
$$

and

$$
\frac{\mathrm{e}^{p(z+4)}(u(z+4)-1)+1}{\mathrm{e}^{p(z)}(u(z)-1)+1}=\frac{w(z+4)}{w(z)}=\frac{R(z+3)}{R(z+1)}=R_{1}(z),
$$

where $R_{1}(z)$ is a rational function. Combining (4.1\}), (4.3) and (4.4), we have

$$
\left(\mathrm{e}^{p(z+4)}-\mathrm{e}^{p(z)}\right) R_{1}(z)(u(z)-1)=\left(1-R_{1}(z)\right)\left(\mathrm{e}^{p(z+4)}-1\right) .
$$

Now, if $\mathrm{e}^{p(z+4)} \not \mathrm{e}^{p(z)}$, then $p \geq 1$ and it follows from (4.5) that

$$
u(z)=\frac{1-R_{1}(z)}{R_{1}(z)} \frac{1-\mathrm{e}^{-p(z+4)}}{1-\mathrm{e}^{p(z)-p(z+4)}}+1 .
$$

Notice that $\operatorname{deg}(p(z)-p(z+4)) \leq p-1$. From (4.6), we can find that

$$
\lambda(u-1)=p>p-1 \geq \rho\left(1-\mathrm{e}^{p(z)-p(z+4)}\right) \geq \lambda\left(\frac{1}{u}\right) .
$$

This is a contradiction to the conclusion of Lemma 4.1. Thus, $\mathrm{e}^{p(z+4)} \equiv \mathrm{e}^{p(z)}$. From (4.2) there exists some integer $k_{1}$ such that

$$
2 k_{1} \pi i=p(z+4)-p(z)=4 p a_{p} z^{p-1}+\cdots,
$$

which yields obviously that $p=1$. Therefore, we see that

$a_{p}=a_{1}=\frac{k_{1}}{2} \pi i$ and hence $p(z)=\frac{k_{1}}{2} \pi i z+a_{0}$ for some constant $a_{0}$.

Case 2: $\boldsymbol{m}=-1$. Now (1.1) is of the form

$$
u(z+1) u(z-1) u(z)=R(z),
$$


which gives

$$
\frac{u(z+3)}{u(z)}=\frac{R(z+2)}{R(z+1)}:=R_{2}(z) .
$$

With this equation and a similar arguing as in Case 1, we can prove that $p(z)=\frac{2 k_{2}}{3} \pi i z+a_{0}$ for some integer $k_{2}$ and some constant $a_{0}$.

Case 3: $\boldsymbol{m}=1$. Now (1.1) is of the form

$$
u(z+1) u(z-1)=R(z) u(z)
$$

which gives

$$
u(z+3) u(z)=R(z+2) R(z+1) .
$$

And hence we have

$$
\frac{u(z+6)}{u(z)}=\frac{R(z+5) R(z+4)}{R(z+2) R(z+1)}:=R_{3}(z) .
$$

It follows this equation that $p(z)=\frac{k_{3}}{3} \pi i z+a_{0}$ for some integer $k_{3}$ and some constant $a_{0}$, and (1.3) holds.

Now, if $w(z)-u(z)$ has a zero $z_{1}$ of multiplicity $\geq 2$ such that $w\left(z_{1}\right)=0$, then from (4.1), we see that $\mathrm{e}^{p\left(z_{1}\right)}=1$.

Rewrite (4.1) as the form

$$
w(z)-1=\mathrm{e}^{p(z)}(u(z)-1) .
$$

Differentiating both sides of the equation above, we have

$$
p^{\prime}(z) \mathrm{e}^{p(z)}(1-u(z))=\mathrm{e}^{p(z)} u^{\prime}(z)-w^{\prime}(z) .
$$

Since $z_{1}$ is a zero of $w(z)-u(z)$ with multiplicity $\geq 2$ such that $w\left(z_{1}\right)=u\left(z_{1}\right)=0$, from the fact that $\mathrm{e}^{p\left(z_{1}\right)}=1$ and (4.7), we find that

$$
p^{\prime}\left(z_{1}\right)=p^{\prime}\left(z_{1}\right) \mathrm{e}^{p\left(z_{1}\right)}\left(1-u\left(z_{1}\right)\right)=\mathrm{e}^{p\left(z_{1}\right)} u^{\prime}\left(z_{1}\right)-w^{\prime}\left(z_{1}\right)=0 .
$$

Thus, $a_{1}=p^{\prime}\left(z_{1}\right)=0$, and hence $\mathrm{e}^{p(z)} \equiv \mathrm{e}^{p\left(z_{1}\right)}=1$. This implies that $w(z) \equiv u(z)$.

Finally, we discuss the Case 2). Since $w\left(z_{j}\right)=u\left(z_{j}\right) \neq 1$ and $z_{2}-z_{3} \notin \mathbb{Q}$, then from (4.1), we can deduce that $\mathrm{e}^{p\left(z_{2}\right)}=1=\mathrm{e}^{p\left(z_{3}\right)}$. Therefore, there exists an integer $k_{0}$ such that

$$
a_{1}\left(z_{2}-z_{3}\right)=p\left(z_{2}\right)-p\left(z_{3}\right)=2 k_{0} \pi i .
$$

If $a_{1} \neq 0$, from the equation above, considering each form of $a_{1}$ for $m=-1,0,1$, we can find that $z_{2}-z_{3}$ must be a nonzero rational number. This contradicts our assumption that $z_{2}-z_{3} \notin \mathbb{Q}$. Thus $a_{1}=0$, and hence $\mathrm{e}^{p(z)} \equiv 1$. This gives $w(z) \equiv u(z)$ again.

\section{Conclusion}

It is shown that the finite order transcendental meromorphic solution of the 
Equation (1.1) is mainly determined by its zeros (or 1-value points) and poles. Examples are provided to show sharpness of our results.

\section{Acknowledgements}

The author is very appreciated for the editors and reviewers for their constructive suggestions and comments for the readability of this paper.

\section{Funding}

This work was supported by the Natural Science Foundation of Guangdong Province (2018A030307062).

\section{Conflicts of Interest}

The author declares no conflicts of interest regarding the publication of this paper.

\section{References}

[1] Laine, I. (1993) Nevanlinna Theory and Complex Differential Equations. In: de Gruyter Studies in Mathematics, Walter de Gruyter, Berlin, New York.

[2] Yang, C.C. and Yi, H.X. (2003) Uniqueness Theory of Meromorphic Functions. Kluwer Academic Publishers, Dordrecht.

[3] Ronkainen, O. (2010) Meromorphic Solutions of Difference Painlevé Equations. Annales Academiae Scientiarum Fennicae Mathematica, 155, 1-59.

[4] Chen, Z.X. (2014) Complex Differences and Difference Equations. Science Press, Beijing.

[5] Cui, N. and Chen, Z.X. (2017) Uniqueness for Meromorphic Solutions Sharing Three Values with a Meromorphic Function to Some Linear Difference Equations. Chinese Annals of Mathematics, Series A, 38, 13-22.

[6] Lü, F., Han, Q. and Lü, W.R. (2016) On Unicity of Meromorphic Solutions to Difference Equations of Malmquist Type. Bulletin of the Australian Mathematical Society, 93, 92-98. https://doi.org/10.1017/S0004972715000787

[7] Zhang, J.L. and Yang, L.Z. (2014) Meromorphic Solutions of Painlevé III Difference Equations. Acta Mathematica Sinica, 57, 181-188.

[8] Lan, S.T. and Chen, Z.X. (2014) On Properties of Meromorphic Solutions of Certain Difference Painlevé Equations. Abstract and Applied Analysis, 2014, Article ID: 208701. https://doi.org/10.1155/2014/208701 